\title{
Hackers Revisited : the "original "hackers"". Clever solutions, political acts.
}

\author{
Camille Akmut
}

\begin{abstract}
We correct here also many details of this literature that has purged the history of modifiers from any political meanings or motivations. We remind of the wider history of systems modifiers.
\end{abstract}




\section{Introduction}

On the significance of the book, it can be said that no other publication has shaped our understanding of the original hackers as Levy's classic Hackers.

Shaping a (new) generation of programmers, computer scientists and indeed "hackers", understanding of who they were, what they did, what they loved, who they loved, what they stood for.

There is no more influential on this topic arguably than Levy's.

This was historically reinforced by Levy's presence and role at Wired, where he wrote regular columns that received much attention. Distributed, first in print, then online, to a specialized, and wide, audience of various - geeks, selfconfessed, self-stylized, programmers, aficionados, hackers in the new sense, hackers in the old sense, hackers in other senses, phone phreaks, freaks short.

We aim to provide here reading notes in the form of commentaries that alternatively enhance, highlight, put into perspective, and offer a critic of this perennial of the "hacker" literature, but more generally, and to come, of computer science history generally.

Hackers. We establish their history, correcting here also dominant uses that obfuscate their true meaning.

Programs are political acts. Modifications are political acts. Political modifications are programs. Political acts are Programs.

\section{The original systems modifiers : clever solu- tions, political acts}

There is no more badly mistreated word in computer technology and science than the word "hacker". Historically associated with some of the most important figures in computer science, primarily around institutions such as MIT, and their various laboratories, it has now been degraded to a state of almost no use, as current and dominant uses of this term, almost all bound with heavy negative connotations, have obfuscated this history, and its original meaning, as well as the practices and philosophies that underpinned it or came with it.

This now largely forgotten part of our history is evoked by Richard Stallman, whom Steven Levy refers to as the "last of the true hackers" in his reference book Hackers, in the following terms :

Hacking included a wide range of activities, from writing software, to practical jokes, to exploring the roofs and tunnels of the MIT campus. Other activities, performed far from MIT and far from 
computers, also fit hackers' idea of what hacking means [e.g. specific musical pieces] (...)

It is hard to write a simple definition of something as varied as hacking, but I think what these activities have in common is playfulness, cleverness, and exploration. Thus, hacking means exploring the limits of what is possible, in a spirit of playful cleverness. ${ }^{1}$

Now associated with fearful imageries, and tales, a "hack" is simply a clever solution in the broadest sense, a playful one in the definition of Stallman.

In all such cases, where the issue of why these transformations take place is concerned, that affect our language, and by extension thoughts, we think of the philosopher and economist Karl Marx's proposal that at any time the dominant ideas are defined/shaped by the dominant groups.

In the specific case of technology, identities such as these pose a direct threat to the dominant groups of our times, which are the large, well-known companies of the technology sector, as their practices put into question their business models - largely based on closed software or hardware systems that cannot be easily repaired or adjusted, or worse in some sense the mass collection and for-profit uses of our private data - and are obstacles to it.

In one striking case, the case of Apple, this company was born out of a well-known hacker community of personal computer modifiers, the Homebrew computer club which operated out of Stanford, of which co-founder Steve Wozniak was an active member, but soon closed off not only their hardware, who count among the hardest to modify, but also their software, itself founded on the hacker culture of UNIX modifiers. ${ }^{2}$ Greenpeace turned into Shell. ${ }^{3}$

We very briefly remind here of this history.

In the 1960s, a rich and colorful community of programmers and systems modifiers started to emerge at universities, in particular at MIT (but also Stanford, and other colleges).

The emergence of the original hackers, or "true hackers" as Levy refers to them, is treated in part one, chapters 1 to 6 , of Hackers (if only one section of this book must be read, it is this one, as it is only a 100 pages long).

In, Hackers, Steven Levy retraces the rich history of the people who, simply

\footnotetext{
${ }^{1}$ Stallman 2002-2019.

${ }^{2}$ More can be found on the Homebrew computer club, including interviews with its original members, in the 1996 PBS documentary Triumph of the Nerds - minus, the critical analysis, as is often the case with such works, which are often celebratory or entertainment in nature. Otherwise, Homebrew is also a subject in Levy's book, chapter 10, "The Homebrew Computer Club" (as part of Part Two. Hardware Hackers).

${ }^{3}$ In similarly striking developments, a large Internet company decided to retract their corporate motto "Don't be evil", while IBM asked from a software developer a "license to do evil", because that developer, Douglas Crockford, had included a note at the very end of the license stipulating "The Software shall be used for Good, not Evil" (JSLint).
} 
put, started to think outside of the box : instead of holding for true that the needs of manufacturers were more important than that of the user, they started to try to find ways to make them [their products] more convenient; improvements that later benefited them. And, if others, had the same problems, they were happy to help them.

companies like Xerox made it a policy to donate their machines and software programs to places where hackers typically congregated. (...) companies could borrow back the improvements, incorporating them into update versions for the commercial marketplace. In corporate terms, hackers were a leveragable community asset, an auxiliary research-and-development division available at minimal cost. ${ }^{4}$

It has been proposed that many programs emerge out of the personal need of the individual programmer. A website may be too intrusive, a printer may not be compatible with one's computer, a mathematics problem, whose calculations are unsolvable by human computation, may find solutions or ways to be moved forward with the help of computers. These needs, in turn, may meet those of other users.

In some instances of the early hacker culture, the division of labor was such that the "theory" part was the domain of some, and the "practical" parts the domain of others : this is to say that some thought the programs, but it befall on others the role and often non-negligible task of actually writing them ${ }^{5}$. History tends to remember the theoreticians, and our society tends to place more value in them, even though without the programmers and hackers these would all only remain in the realm of pleasant or Platonic ideas, or fictions of the cultivated mind. ${ }^{6}$

Kotok's role in bringing the chess program to life was indicative of what was to become the hacker role in Artificial Intelligence: a Heavy Head like McCarthy or his colleague Marvin Minsky would begin a project or wonder aloud whether something might be possible, and the hackers, if it interested them, would set about doing it. ${ }^{7}$

Much of the (early) history of computing at universities, and much of the history of hackers, is accompanied by such Maussian-like gifts (here called neu-

\footnotetext{
${ }^{4}$ Williams 2002, Chapter 1.

${ }^{5}$ For these thinkers may not actually be able to do it by themselves, either partly or entirely (McCarthy had started writing it, the chess program).

${ }^{6}$ In the following quote, Kotok is Alan Kotok: "MIT student from Jersey who worked under the rail layout at TMRC [Tech Model Railroad Club], (...) a legendary TX-0 and PDP-1 hacker." (Hackers, "Who's Who", p. xiii)

${ }^{7}$ Levy $2010: 36$.
} 
trally "donations") : free equipment, then extremely $\operatorname{costly}^{8}$, in exchange for labor, now much more costly ${ }^{9}$.

The original culture of hackers had emerged at MIT. In a second era, it then moved to other places, notably Stanford ${ }^{10}$.

At Stanford, the hacker culture that had emerged at MIT was transplanted as prominent figures within that culture moved from one institution to the other, a phenomenon that was often of key importance for the transfer and communication of ideas and practices in pre-Internet and -Web eras ${ }^{11}$.

Levy describes it as "the closest thing to the MIT hacker community [but] only an approximation of the ideal" ${ }^{12}$.

But the California distortion was a significant one, demonstrating how even the closest thing to the MIT hacker community was only an approximation of the ideal; the hothouse MIT style of hackerism was destined to travel, but when exposed to things like California sunlight it faded a bit in intensity.

Similar developments at Berkeley are discussed in Chapter 8 of Hackers, "Revolt in $2100 "$.

In the documentary film The Code: Story of Linux, Eric Raymond claims : "I use the word hacker in its correct, and original sense, to describe a person who pursues computer programming as a kind of artistic passion, and who also is part of, or identifies with the "hacker culture", which is a group of programmers, historically, that has produced the Internet, and Linux, and the World Wide Web." This is both correct and mostly incorrect.

Many hackers conceived of programming as either a political practice or a political act, were aware of the political or social implications, or had otherwise a strong political background. This is the case of Lee Felsenstein (described at length in chapter 8 of Hackers), Richard Stallman (the entirety of part four of the same), and elsewhere we have given indications of McCarthy's social background (extraordinarily downplayed in Hackers and almost all publications), whose parents had strong roots in the Communist party, labor organization,

\footnotetext{
${ }^{8}$ The TX-0 cost 3 million then-dollars (Levy $2010: 14$ ).

${ }^{9}$ In domains such as Artificial Intelligence, salaries for personnel of the qualifications described in Hackers would be in the range of about a quarter to a million dollars per year currently (approximations derived from Sample 2017, Bloomberg 2017).

${ }^{10}$ Developments at Stanford start to be evoked from Chapter 7 of Hackers onward.

11 "In a development that was to have considerable impact on spreading MIT-style hackerism outside of Cambridge, John McCarthy left the Institute to begin a new artificial intelligence lab on the West Coast, at Stanford University. Slug Russell, ever McCarthys LISP-writing coolie, tagged along." (Levy 2010 : 56) The laboratory mentioned is SAIL (Stanford Artificial Intelligence Laboratory, the Stanford AI Lab) whose current-day equivalent at MIT is CSAIL, formed out of the merger of the CS and AI Lab's.

${ }^{12}$ Levy $2010: 134$.
} 
publishing and social work; roots that may shed light on, if not explain the philosophical-political aims he attached to artificial intelligence.

These people were the planners. Among them were scientists who occasionally engaged in hacking-Jack Dennis, McCarthy, Minskybut who were ultimately more absorbed by the goals of computing than addicted to the computing process. They saw computers as a means to a better life for the human race, but did not necessarily think that working on a computer would be the key element in making that life better. Some of the planners envisioned a day when artificially intelligent computers would relieve man's mental burdens, much as indus- trial machinery had already partially lifted his physical yoke. ${ }^{13}$

We are not aware of Berners-Lee specifically identifying with the hacker culture described here, but may be wrong (he grew up and spent most of is life in England, then in Switzerland, where it is unclear how these ideas would have channeled over there), while Linus Torvalds grew up in an even more geographically remote part of Europe, Finland, and we are also not aware of him identifying as a "hacker", though his practices may overlap with theirs.

In any case, in the "original sense", we understand hackers to have originated at MIT around the modification of various early large computers (the TX-0 then PDP-1 in particular). ${ }^{14}$

In general, these political elements are downplayed, painting the image of the timid or aloof "geek", which sometimes may meet reality, but sometimes not. This is also the case in Hackers.

things that the hackers did not talk about. They did not spend much time discussing the social and political implications of computers in society (except maybe to mention how utterly wrong and naive the popular conception of computers was). They did not talk sports. They generally kept their own emotional and personal lives-as far as they had any-to themselves. And for a group of healthy collegeage males, there was remarkably little discussion of a topic, which commonly obsesses groups of that composition: females. ${ }^{15}$

${ }^{14}$ Raymond is most likely aware of all of this, having taken upon himself to edit a preexisting dictionary of these topics, and the reason why he may omit certain parts of history is that mentioning the hackers of MIT, would mean mentioning the last true of the hackers, from whom he is separated by countless ideological-political-philosophical oppositions, that are then found again, but transformed, in countless polite discussions and seemingly neutral presentations, saids and un-said. But : liberties, everywhere but in history, and the language that we use, which, preceding computers by far, though influencing our relationship with them, is our primary tool, of incidence on almost everything that we do - and are able to do. ${ }^{15}$ P. 74.
} 
Seemingly contradicting himself in following developments (mentioned above).

This depiction, though part of the 2010 reissue, also displays a largely heteronormative view of the world, a world seemingly stuck where Levy's narrative choices are concerned in 1990's male locker rooms, Don't ask, don't tell politics, Friends, and Beer and peanuts, and NBA-finals-wit'-the-pals. Perhaps the author ought to open up his world a little bit.

He shows an extraordinary lack of attention to anything outside of his own well-delimited world in the case of non-heterosexuality. Does Levy mean to say that none of the Hackers described over the over 500 pages of his book were gay or bisexual, or otherwise queer or asexual? This seems hard to believe. If it were the case however, and it is not, it would mean Levy would have - rare if ever the case among historians, journalists and others - gained very intimate insights on a very broad group of individuals, and his work would be almost unique in this. ${ }^{16}$

To say nothing of the absence of women. Who are the well-known female hackers? We won't know reading Levy's book, and such a history also remains to be written.

In some non-negligible way, in Hackers, Levy both describes and constructs his subject - the hallmark of a great book, and it is one, but not of a good historical account. The signs of which are usually given when a book reaches a notoriety or status that outshadows its subjects.

Propositions :

- Maybe some of them didn't talk about girls, because they weren't interested in them (for reasons specifically others than their engrossment with computers) i.e. because they liked men better. Hypothesis 1 .

- Maybe some of them didn't talk about girls, because they couldn't talk about their actual preferences, see hypothesis 1 , because the period that the author writes about, early 1960's onward, was one marked by a combination of legal, political and social stigmatization, repression and criminalization of homosexuality. And, maybe, because among their peer groups, they had people like Levy who thought, in their youthful naivety and enthusiasm, that their world was the only one that existed, and by doing so imposed their values onto others, making it harder for those others if not impossible to talk about their own things. Hypotheses 2.

\footnotetext{
${ }^{16}$ The existence and lives of the well-known UNIX programmers, if not hackers, out of Berkeley, Marshall McKusick, and his partner, Eric Allman, alone contradict this. For a future edition, if one is planned, he should simply reach back to some of his interviewees and ask, and his story would be much richer for it : in ways he would almost certainly be surprised by, offering other insights into other things at the same time. In this hypothetical edition, gay would appear in passages other than to mean happy, in the old sense.
} 
- If female hackers had been taken into account, maybe some of them didn't talk about men, because they preferred the company of women instead, including romantically, because they hadn't cared a day in their lives for men - a seemingly infinite source of surprises and pondering for some men. Hypotheses 3.

- Hypotheses 2 applied to women. Hypotheses 4.

- Hypotheses 5 : taken into account the degree of educational segregation of women in STEM subjects during that period, what places or locations could have seen the emergence of a female hacker culture? E.g. at MIT, we know that the construction of McCormick Hall was an important event (1963 West Tower; extended in 1967 through East Tower) : for the first time women had housing on the MIT campus other than in an ad hoc, temporary or uncertain fashion. Who are the hackers of McCormick Hall? We don't know, McCormick Hall never appears in Hackers.

- Hypotheses 6 : we know from other periods in history that exclusively male cultures ${ }^{17}$, with almost no failure, unless specifically repressed or undermined, lead to the development of rich homosexual or pseudo-homosexual cultures, of the pederastic, pseudo-pederastic, egalitarian, or modern egalitarian kind $^{18}$. Historically, this could have been because a society had adopted a model that recognized only men with full rights or status (Ancient Greece, specifically Athens ${ }^{19}$ ), and/or men-men relationships were celebrated in culture and art, philosophy, literature and vase paintings for instance (Plato's two dialogues, Achilles and Patroclus, various vases including representations of the former), or because of the existence of religious-monastic institutions, and/or societies where emperors and the new merchant class engaged in such practices (Japan, China), or, more controversially because of the uneven ratio of women and men in some urban areas; or, indeed, because of all-male educational cultures, in particular when these include the feature of, equally gender-exclusive, on-campus housing ${ }^{20}$.

A portrait or reality most friendly and agreeable to corporations whose various infantilizing "perks" appeal to them.

\footnotetext{
17 "And they formed an exclusively male culture." (Levy 2010 : 76).

${ }^{18}$ Dover, Greek Homosexuality. Leupp, Male Colors: The Construction of Homosexuality in Tokugawa Japan.

${ }^{19}$ It is often incorrectly stated or assumed that this model was valid in all of Ancient Greece. Whenever we talk of Ancient Greek homosexuality, we most of the time mean the specific model of pederasty that existed in Athens. In other cities and islands, Dover suggests, other models including of the modern or egalitarian kind may have existed - lack of sources.

${ }^{20}$ Described, in the cases of Oxford and Cambridge, in the first half of Brideshead Revisited and Maurice, by E. M. Forster.
} 


\subsection{Printer modification by Richard Stallman}

One example of a particular modification is given by a modification realized by the then young and unknown computer scientist Richard Stallman.

Richard Stallman is described by Levy as famously "The Last of the True Hackers". His portrayal closes the book ${ }^{21}$.

The thing he liked about the AI lab at Tech Square was that "there were no artificial obstacles, things that are insisted upon that make it hard for people to get any work donethings like bureaucracy, security, refusals to share with other people." He also loved being with people for whom hacking was a way of life. He recognized that his personality was unyielding to the give-and-take of common human interaction.

In many passages, the clash between the old culture of freedom - no passwords hence no separation of files into private and public files on public computers hence also no possibilities to create ownership - and the new demographics that entered the institutions that had been associated with the hacker culture of old are evoked.

He encouraged people to use the "Empty String" password-a carriage return instead of a word. So when the machine asked for your password, you would hit the RETURN key and be logged on.

$(\ldots)$

[It] eventually got to a point where a fifth of all the users on the machine had the Empty String password

$(\ldots)$

Now, some of these new users could not handle the freedom to poke around a system with everyones files open to them.

$(\ldots)$

Like Third-Generation hackers, they saw nothing wrong with the concept of ownership of programs.

We focus on one particular episode of a hack, a printer modification ${ }^{22}$. We will simply refer to this program as Printer modification.

Having noticed that a printer did not work according to the users' needs, a distinction of importance as it implied that there were such needs and that they were or could be different or differ from those of the manufacturers, in this case the occurrence of paper jams, he developed an alternative (or modification).

\footnotetext{
${ }^{21}$ Levy, Hackers, "Part Four. The Last of the True Hackers".

${ }^{22}$ This episode is the focus of the opening chapter of Sam Williams' Free as in Freedom.
} 
Chief among the drawbacks was the machine's inherent susceptibility to paper jams. Engineering-minded programmers quickly understood the reason behind the flaw. As a photocopier, the machine generally required the direct oversight of a human operator. Figuring that these human operators would always be on hand to fix a paper jam, if it occurred, Xerox engineers had devoted their time and energies to eliminating other pesky problems. In engineering terms, user diligence was built into the system. ${ }^{23}$

He had already encountered a similar problem in another printer, he applied the same fix to it, by adding new lines (a new feature) to the software: when a jam occurred, a message should, and would be sent out to those who intended to use the printer, so that they could fix it.

As fixes go, Stallman's was oblique but elegant. It didn't fix the mechanical side of the problem, but it did the next best thing by closing the information loop between user and machine. ${ }^{24}$

Hacks often are not perfect, or direct, but they try to find some way to fix a problem. Newer, better solutions, can then always improve on it, by providing more convenience (e.g. the printer should fix the problem by itself as much as possible), but at least one solution was there. This, of course, requires the necessity for code, and knowledge, to be openly viewable (accessible) and (freely) sharable. ${ }^{25}$

\section{Conclusion}

We corrected the a-political and heteronormative parts of Levy's narration, and reestablished a history of systems modifiers were political elements were not absent.

Foucault wrote that he wrote to change the way he thought. We write to change a discipline so that it will be pleasant to be a part of it - on top of all the good things it all already has. The cake and cherries - no beer and peanuts for us.

Hackers, "in the new sense", will mean all the queers and the weirdos, and the queer weirdos. Those who do interesting things with computer. Interesthings. "Programs that make us dream".

\footnotetext{
${ }^{23}$ Ibid.

${ }^{24}$ Ibid.

${ }^{25}$ Richard Stallman then went on to organize GNU, a free operating system, an alternative to the dominant model of closed such systems, and free licenses called GPL (General Public License), which have been used by Linux and other projects; operating simultaneously a modification on the system of such systems, and systems other than these systems.
} 


\section{Bibliography}

Bloomberg News. 2017. "This Startup Is Luring Top Talent With $\$ 3$ Million Pay Packages". 24/09.

https://www.bloomberg.com/news/articles/2017-09-24/in-battle-for-talentone-startup-founder-tries-unlimited-pay

Huang, Andrew. 2018. "Making \& Breaking Hardware: Origins of the Hacker Ethos". https://www. youtube. com/watch?v=HwkbbVoQOxw

Levy, Steven. 2010. Hackers: Heroes of the Computer Revolution. Sebastopol, CA : O'Reilly Media.

Sample, Ian. 2017. "Big tech firms' AI hiring frenzy leads to brain drain at UK universities". The Guardian, 02/11.

Stallman, Richard. 2002-2019. "On Hacking". https://www.stallman.org/ articles/on-hacking.html.

Williams, Sam. 2002. Free as in Freedom: Richard Stallman's Crusade for Free Software. Sebastopol, CA : O'Reilly Media.

https://www . oreilly.com/openbook/freedom/ 